\title{
The Longest-Lasting Relationship: Patterns of Contact and Well- Being Among Mid- to Later-Life Siblings
}

\author{
Alexander C. Jensen \\ Brigham Young University - Provo, alexjensen@byu.edu \\ Makayla K. Nielson \\ Brigham Young University - Provo \\ Jeremy B. Yorgason \\ Brigham Young University - Provo
}

Follow this and additional works at: https://scholarsarchive.byu.edu/facpub

Part of the Other Social and Behavioral Sciences Commons

\section{Original Publication Citation}

Jensen, A. C., *Nielson, M. K., \& Yorgason, J. B. (2019). The longest lasting relationship: Patterns of contact and well-being among mid to later life siblings. The Journals of Gerontology: Series B.

\section{BYU ScholarsArchive Citation}

Jensen, Alexander C.; Nielson, Makayla K.; and Yorgason, Jeremy B., "The Longest-Lasting Relationship: Patterns of Contact and Well-Being Among Mid- to Later-Life Siblings" (2019). Faculty Publications. 4147. https://scholarsarchive.byu.edu/facpub/4147

This Peer-Reviewed Article is brought to you for free and open access by BYU ScholarsArchive. It has been accepted for inclusion in Faculty Publications by an authorized administrator of BYU ScholarsArchive. For more information, please contact ellen_amatangelo@byu.edu. 


\title{
The Longest-Lasting Relationship: Patterns of Contact and Well-Being Among Mid- to Later-Life Siblings
}

\author{
Alexander C. Jensen, PhD, ${ }^{*,}$ Makayla K. Nielson, MS, and Jeremy B. Yorgason, PhD \\ School of Family Life, Brigham Young University, Provo, Utah. \\ *Address correspondence to: Alexander C. Jensen, PhD, Brigham Young University, Provo, UT 84604. E-mail: alexjensen@byu.edu
}

Received: July 23, 2018; Editorial Decision Date: June 5, 2019

Decision Editor: J. Jill Suitor, PhD

\begin{abstract}
Objectives: Adults in mid to later life experience shrinking social networks, which may hinder well-being. Siblings may be important sources of social contact. Yet, little is known about adults' patterns of contact with siblings and how contact is linked to well-being.

Method: Participants included 491 adults from across the United States ( $M$ age $=58.96, S D=6.25 ; 68 \%$ female) recruited online via Amazon Mechanical Turk; they reported on their contact with their sibling in person, over the phone, via email, texting, and social media.

Results: Latent class analysis found evidence for four patterns of contact (classes) among siblings: low, medium, high, and traditional. Those with high contact reported greater life satisfaction than those in the other groups. Those in the high group reported lower self-rated health when they recalled being treated less favorably, relative to their sibling, by their mother as children.

Discussion: These findings suggest that differing patterns of sibling contact exist among older adults. In some cases, contact may promote well-being. In other cases, more contact may serve as a reminder of hurtful or painful past family experiences related to mothers' differential treatment, in which case more contact may be linked to poorer health.
\end{abstract}

Keywords: Contact, Life satisfaction, Parental differential treatment, Self-rated health, Siblings

The Baby Boomer generation is rapidly growing older as "roughly 10,000 Baby Boomers will turn 65 today, and about 10,000 more will cross that threshold every day" until 2030, at which point nearly $20 \%$ of Americans will be older than 65 (Cohn \& Taylor, 2010). Many of these adults will be at risk for lower well-being (Gray, 2009; Litwin, \& Stoeckel, 2013), but previous research suggests that social contact can promote well-being (Pinquart \& Sorensen, 2000); contact with siblings may be uniquely important (Bedford \& Avioli, 2012). Little is known, however, about levels and patterns of contact among older siblings and if contact is linked to well-being. Using a Life Course perspective, the goals of the current paper were to examine patterns of contact among siblings in mid to later life and to explore implications for well-being.

\section{Patterns of Contact}

A critical component of Life Course Theory is that transitions impact development and relationships, and families' lives are linked in a way that members' experiences influence others in their family (Bengston, Elder, \& Putney, 2005; Elder, Johnson, \& Crosnoe, 2003). Life transitions may pull siblings apart and bring them back together, but their lives are always interconnected. For example, at younger ages, siblings spend more time together than they do with other family members (Feinberg, Solmeyer, \& McHale, 2012). Moving into young adulthood siblings tend to leave their family of origin and experience decreased contact (White, 2001) and changes in relationship quality (Jensen, Whiteman, \& Fingerman, 2018). Further into adulthood, 
individuals become busy with their own lives and families, which may further diminish siblings' connections (Milevsky, 2005).

Moving into mid and later life, individuals go through more transitions that may allow for a reemergence of sibling relationships and contact (White, 2001). The launching of children, transitioning out of working life, and caring for aging parents may create more time to communicate with a sibling than in the previous years of life. According to Life Course Theory (Bengston et al., 2005; Elder et al., 2003), these major transitions affect the trajectories of social relationships of the individual and because of past shared familial experiences, these transitions may promote a reemergence of sibling contact during this stage of life.

Life Course Theory also suggests that historical time plays a role in the individual's life course (Bengston et al., 2005), and Baby Boomers live in a time different from previous decades. For years, in person, mail, and telephone contact were the main options for sibling contact in mid to later life. The mid 1990s brought major change in communication, when many people started using email. In the early 2000s texting started to become more common. In 2006, Facebook and Twitter became available to the public, and Instagram in 2010 . Current data suggests that $80 \%$ of those aged 65 years and older own a cell phone, $64 \%$ are internet users, and $34 \%$ have one or more social media accounts (Pew Research Center, 2017). Given that many Baby Boomers are under 65 years, the rates for all older adults of the Baby Boomer generation and beyond are likely higher. Thus, many older siblings may keep in contact via a combination of mediums.

Because siblings likely keep contact with one another through a variety of ways, it is important to consider patterns of contact. Some siblings may maintain frequent contact through multiple methods and others may have very little contact, regardless of medium. Others may keep in touch in more traditional ways, or mainly through technological means. Given the dearth of research on this topic, our first aim was to assess the types of patterns and prevalence of contact among older adult siblings via in person visits, phone calls, emailing, texting, and the use of social media.

\section{Links with Well-Being}

According to Life Course Theory (Bengston et al., 2005; Elder et al., 2003), the transitions mid to later life adults experience, including the launching of their grown children (Siordia et al., 2017), retirement (Munnell, 2015), and caring for aging parents (Halliwell, Wenzel Egan, \& Howard, 2017) impact development and well-being. For example, current literature suggests that these adults are at greater risk for low well-being than at younger ages (Gray, 2009; Litwin, \& Stoeckel, 2013) and social contact is critical to promoting well-being among mid- to later-life adults (Pinquart \& Sorensen, 2000). Because of their past shared familial experiences, contact with siblings may play an important role in promoting well-being (Bedford, 1989; Liao \& Carstensen, 2018).

The link between contact and well-being may vary by patterns of contact between siblings. Recent work suggests that face-to-face contact is particularly important for older adults in combating loneliness (Teo et al., 2015). Other research, suggests that communication via technological means can have positive effects for older adults (Ferreira, Torres, Mealha, \& Velso, 2015). Given our question about patterns of contact we did not hypothesize about difference among specific groups but we explored whether groups based on differing types of mediums were differentially linked to well-being.

According to Life Course Theory, early experiences in life may have implications for future development and relationships (Elder et al., 2003) via a series of cumulative continuities (Caspi, Elder, \& Bem, 1988). Aspects from childhood sibling processes may moderate links between contact and well-being in later life. In the current study, we focused on parental differential treatment (PDT) because its implications across the life-course (Gilligan, Suitor, Kim, \& Pillemer, 2013; Scholte, Engels, de Kemp, Harakeh, \& Overbeek, 2007). There is debate, however, as to the role PDT plays. Some studies suggest that differences in treatment in adulthood have negative implications for adults, regardless of favored status (Boll, Ferring, \& Filipp, 2003; Suitor et al., 2009). This approach is labeled absolute PDT because it contrasts equal treatment with the absolute amount of differences in treatment, and is typically linked to familial relationships. Other research uses a relative PDT perspective, which contrasts favored and less-favored status and suggests differing effects. For example in midlife less-favored siblings may report lower well-being (Suitor, Gilligan, Peng, Jung, \& Pillemer, 2017), and in some cases, favored siblings may report better well-being (Jensen, Whiteman, Fingerman, \& Birditt, 2013). In the current study, we used the relative PDT approach because of its consistent links with well-being.

Although concurrent differences in treatment are salient in adulthood (Gilligan et al., 2013; Jensen et al., 2013; Suitor et al., 2017), past work suggests that recollections of PDT from childhood are also important (Davey, Tucker, Fingerman, \& Savla, 2009; Peng, Suitor, \& Gilligan, 2018; Suitor et al., 2009). Thus, in line with the notion from Life Course Theory (Bengston et al., 2005; Elder et al., 2003) that early life experiences can play a role in future development, we focused on how recollections of PDT from childhood are linked with sibling processes in later life. When adults have higher levels of contact with a sibling, the link with well-being may be positive or negative, depending on their recollection of childhood PDT. On the one hand, for older adults who believe they received favored parental treatment in childhood, regular contact with a sibling may be a consistent reminder of that favored status; thus, more contact may promote well-being. On the other 
hand, for older adults who believe they were disfavored as a child, higher levels of contact with a sibling may serve as a reminder of the disfavored status; thus, more contact may hinder well-being. Past work has suggested that PDT from mothers and fathers have differing effects (Gilligan et al., 2013; Jensen et al., 2013), thus, we examined PDT of mothers and fathers separately. Additionally, because the goal of the current study was not focused on deeper nuances of differences in treatment, we chose to focus on overall differences based on a number of domains to achieve a broader view of recalled differences.

\section{Present Study}

Rooted in Life Course Theory (Elder et al., 2003) and sibling research (Gilligan et al., 2013; White, 2001), the current study examined patterns of contact among siblings in mid to later life and how those patterns were linked to well-being. We included separate reports of contact via in person, telephone, email, texting, and social media. We hypothesized that patterns marked by more contact would be linked to better well-being, especially for those who recalled favored treatment in childhood. For those who recalled less-favored treatment in childhood, we hypothesized that patterns with higher levels of contact would be linked to lower well-being. We examined two aspects of well-being, life satisfaction and self-reported physical health. Additionally, we controlled for siblings' overall closeness and conflict so that observed effects regarding patterns of contact would reflect better estimates of the role of contact not conflated with relationship quality.

\section{Method}

\section{Participants}

Participants were drawn from Amazon Mechanical Turk (MTurk). MTurk, is an online collection of "workers" available to complete online tasks, including surveys (Buhrmester, Kwang, \& Gosling, 2011). MTurk has over 500,000 potential participants, and roughly $20 \%$ of MTurk workers were born before 1965 (Ipeirotis, 2010). Although MTurk is a convenience sample, it provides a more diverse sample than traditional internet or convenience sampling, and previous work using test-retest reliability has found participants to provide reliable data (Buhrmester et al., 2011; Rouse, 2015).

Data were limited to participants born before 1965 (i.e., members of the Baby Boomer generation or older), which had at least one living sibling, and resided in the United States. The final sample included 491 adults who each reported on their closest aged living sibling. Participants came from every state except Vermont and Alaska, and their siblings resided in every state except Vermont. Demographic information for participants and their closest aged sibling are presented in Table 1 . Although samples on
Table 1. Demographic Characteristics of Participants and Their Closest Aged Sibling $(N=491)$

\begin{tabular}{|c|c|c|}
\hline & Participant & Sibling \\
\hline Variable & $\begin{array}{l}M(S D) \text { or } \\
\text { Proportion }\end{array}$ & $\begin{array}{l}M(S D) \text { or } \\
\text { Proportion }\end{array}$ \\
\hline Age & $58.96(6.25)$ & $58.73(8.78)$ \\
\hline Income $^{\mathrm{a}}$ & $4.60(2.38)$ & $5.40(2.78)$ \\
\hline $\begin{array}{l}\text { Number of living } \\
\text { siblings }\end{array}$ & $2.74(1.79)$ & $2.74(1.79)$ \\
\hline Distance from sibling ${ }^{\mathrm{b}}$ & $5.00(2.56)$ & $5.00(2.56)$ \\
\hline Women & .68 & .49 \\
\hline Married & .56 & .62 \\
\hline Has children & .78 & .73 \\
\hline Full biological siblings & .92 & .92 \\
\hline \multicolumn{3}{|l|}{ Birth order } \\
\hline Firstborn & .37 & .30 \\
\hline Middleborn & .36 & .39 \\
\hline Lastborn & .27 & .32 \\
\hline \multicolumn{3}{|l|}{ Level of education } \\
\hline Advanced degree & .18 & .13 \\
\hline Bachelor's degree & .33 & .29 \\
\hline Some college & .36 & .26 \\
\hline High school & .11 & .24 \\
\hline \multicolumn{3}{|l|}{ Employment status } \\
\hline Full time & .38 & .52 \\
\hline Part time & .19 & .10 \\
\hline Retired & .24 & .21 \\
\hline \multicolumn{3}{|l|}{ Race/Ethnicity } \\
\hline White & .87 & .87 \\
\hline Non-White & .13 & .13 \\
\hline Mother living & .48 & .48 \\
\hline Father living & .27 & .27 \\
\hline
\end{tabular}

Note: ${ }^{\mathrm{a}} 1=$ Less than $\$ 10,000,2=\$ 10,001-\$ 25,000,3=\$ 25,001-\$ 40,000$, $4=\$ 40,001-\$ 50,000,5=\$ 50,001-\$ 60,000,6=\$ 60,001-\$ 75,000$, $7=\$ 75,001-\$ 100,000,8=\$ 100,001-\$ 125,000,9=\$ 125,001-\$ 150,000$, $11=\$ 150,001-\$ 200,000,12=\$ 200,001-\$ 250,000,13=\$ 250,001$ or more. ${ }^{\mathrm{b}} 1$ = same household $; 2=0-25 ; 3=26-50 ; 4=50-100 ; 5=101-250 ; 6=251-$ $500 ; 7=501-1,000 ; 8=1,001-2,000 ; 9=2,000+$ miles.

MTurk are generally more diverse than other convenience samples (Buhrmester et al., 2011; Casler, Bickel, \& Hackett, 2013), we compared the sample for this study to a larger national data set (the CORE cohort of the 2016 Health and Retirement Study [HRS]) to examine differences from the general population. Compared to the HRS, participants in the current study were younger $(t=15.46, d f=20,325, p$ $<.001)$, more educated $\left(X^{2}=247.88, d f=4, p<.001\right)$, less likely to have children $\left(X^{2}=247.88, d f=4, p<.001\right)$, more likely to be Caucasian/White $\left(X^{2}=247.88, d f=4, p<.001\right)$, more likely to be female $\left(X^{2}=247.88, d f=4, p<.001\right)$, more likely to be employed $\left(X^{2}=247.88, d f=4, p<.001\right)$, and more likely to have had their mother $\left(X^{2}=247.88\right.$, $d f=4, p<.001)$ and father pass away $\left(X^{2}=247.88, d f=4\right.$, $p<.001)$. They did not differ in marital status or median income. 


\section{Procedure}

The survey was administered online and took approximately $18 \mathrm{~min}$ to complete. Consistent with other studies on MTurk (Bohannon, 2011), participants were given an honorarium of $\$ 0.50$ for completing the survey. All survey sections were presented in a randomized order, and all items within each measure were also presented in a randomized order. Internet samples are at risk for inattentive participants who produce inaccurate data, we took two critical steps to ensure honest and quality data. MTurk workers each have a score of the percentage of tasks they have accurately completed. We restricted participants to those who had a 95\% approval or higher (Casler et al., 2013). Additionally, two attention checking questions were included (e.g., I have been to every country on earth) to track inattentive participants (Rouse, 2015). Those who answered attention checking questions incorrectly were excluded $(n=5)$. All procedures were approved by the Institutional Review Board of the first author's university (protocol X15397).

\section{Measures}

\section{Sibling contact}

Participants reported on sibling contact using five items adapted from Silverstein, Gans, and Yang (2006). Based on a nine-point scale from 1 (never), 3 (once a year), 5 (monthly), 7 (weekly), to 9 (daily), participants rated their frequency of contact with their sibling in person, over the phone, via email, via text messaging, and through social media.

\section{Recalled parental differential treatment}

Participants reported on recalled parental differential treatment from their childhood from mothers and fathers. The measure included four items adapted from the Sibling Inventory of Differential Experiences (Daniels \& Plomin, 1985). Items assessed recollection of who received preferential treatment in terms of conflict, affection, time, and overall treatment. The items were assessed on a fivepoint scale from 1 (me a lot more), 3 (both of us the same), to 5 (my sibling a lot more), and showed adequate reliability for mothers $(\alpha=.79)$ and fathers $(\alpha=.76)$. For analysis, items were centered at equal treatment.

\section{Life satisfaction}

Participants reported on one item from Diener, Gohm, Suh, and Oishi (2000) on a 10-point scale ranging from 1 (not at all satisfied) to 10 (completely satisfied). The item was worded, "How satisfied are you with your life overall?"

\section{Self-rated health}

Participants rated their health using one item taken from Mossey and Shapiro (1982). The item was on a five-point scale ranging from 1 (poor), 3 (good), to 5 (excellent).
The item was worded, "During the past 12 months, in general, would you say your physical health has been?"

\section{Control Variables}

\section{Sibling closeness}

Participants reported on sibling closeness using five items from Blyth and Foster-Clark (1987). Items were based on a five-point scale from 1 (never), 3 (sometimes), to 5 (very often). Example items included, "How often do you go to each other for advice or support?" and "How often do you accept each other no matter what you do?" All items were averaged together $(M=3.35, S D=1.05$, $\alpha=.88)$.

\section{Sibling conflict}

Participants reported on sibling conflict using three items adapted from Stocker and McHale (1992). Items were based on a five-point scale from 1 (not at all), 3 (some), to 5 (very much). Participants were asked, "Thinking of your relationship with [sibling name], how much do you and [sibling name]. .." Items included, "Get upset or mad at each other?", "Get annoyed with each other?," and, "Argue with each other." Items were averaged together $(M=2.19$, $S D=.97, \alpha=.92$ ).

\section{Results}

At the bivariate level (Supplementary Table 1), all types of contact were positively associated with one another. Each type of contact was positively correlated with life satisfaction, although correlations were small. Frequency of texting was positively associated with self-rated health.

There were no missing data on the variables used in the study.

\section{Patterns of Contact}

We used Mplus Version 8 (Muthen \& Muthen, 2017) to conduct a latent class analysis of sibling communication profiles based on the mediums of contact (in person, telephone, email, text, and social media). Relative model fit statistics, the Vuong-Lo-Mendell-Rubin (VLMR) test, and entropy scores were used to determine the best number of classes. The four-class solution best represented the data (Supplementary Table 2), with the -2 Log Likelihood (-2LL), Akaike Information Criterion, Bayesian Information Criterion, and Quality of Numerical Results decreasing from the three-class solution, and the VLMR test being statistically different from zero. In contrast, the five-class solution had a VLMR value that was not different from zero. The four-class solution was further supported by having classes comprised of $16.5 \%$ or more of the sample in each class. Detailed fit statistics can be found in the Supplementary Materials. 
As shown in Figure 1, a plot of the four-class solution, the first, third, and fourth classes represent respondents who reported to communicate at low, medium, and high rates, respectively. The second class of respondents differed in that they typically communicated via more traditional methods (in person or by phone). Class membership size was as following: low communication $(n=164)$, medium communication $(n=164)$, high communication $(n=81)$, and traditional communication $(n=82)$. Although not used in our analysis, the five-class solution retained the high, low, and traditional groups but split the medium group into medium-high and medium-low.

We conducted post hoc analyses to examine ways our findings may vary based on the areas our sample differs from the general population. First, we conducted a series of Ordinary Least Squares (OLS) Regression models to examine how age, years of education, parental status $(0=$ no children; $1=1+$ children $)$, employment status $(0=$ not employed; 1 = employed full or part time), if the mother was living $(0=$ not living; $1=$ living $)$, and if the father was living $(0=$ not living; 1 = living $)$ were associated with each type of contact. In person contact was negatively associated with years of education $(b=-0.37, S E=.10, p<.001, \beta=-.17)$, positively associated with employment status $(b=0.49$, $S E=.20, p<.05, \beta=.11)$ and positively associated with the fathers' living status $(b=0.53, S E=.24, p<.05, \beta=.11)$. Texting was positively associated with employment status $(b=0.90, S E=.24, p<.001, \beta=.17)$ and the fathers' living status $(b=1.07, S E=.29, p<.001, \beta=.18)$. Social media contact was negatively associated with education $(b=-0.25, S E=.12, p<.05, \beta=-.09)$ and positively associated with employment status $(b=0.70, S E=.25, p<.01$, $\beta=.13)$. Additionally, using multinomial logistic regression (low communication class as the reference group) we examined whether class membership was predicted by the areas our sample differed from the general population. We found that older participants were more likely to be in the traditional class (odds ratio $[\mathrm{OR}]=1.07,95 \%$ confidence interval [CI], 1.02-1.12). Participants who were more educated were less likely to be in the high communication class $(\mathrm{OR}=0.64,95 \% \mathrm{CI}, 0.48-0.85)$, whereas those who were employed $(\mathrm{OR}=2.44,95 \% \mathrm{CI}, 1.33-4.47)$ or had a living

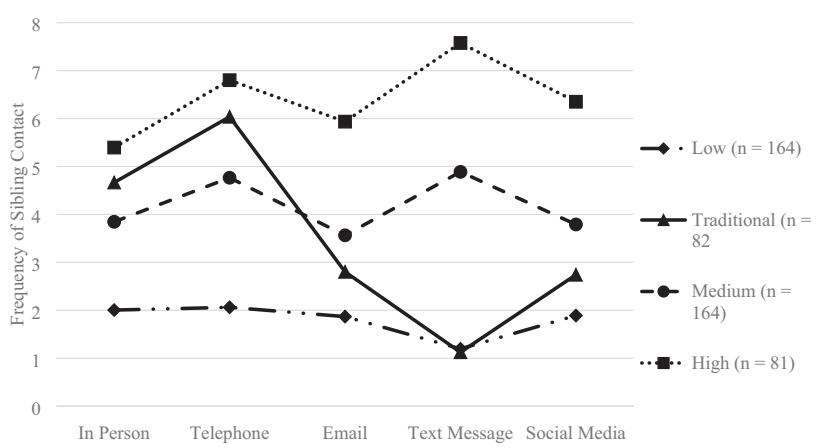

Figure 1. Plot of latent classes for the four-class solution of sibling communication. father $(\mathrm{OR}=2.59,95 \% \mathrm{CI}, 1.32-5.11)$ were more likely to be in the high communication class.

\section{Links with Well-Being}

Links with well-being were tested in two sets of OLS models (one set for each dependent variable). The sets were tested hierarchically in identical fashion. In each set, in the first step, we entered if the mother was living $(0=$ not living; $1=$ living $)$, if the father was living $(0=$ not living; $1=$ living $)$, if parents ever divorced $(0=$ no; $1=$ yes $)$, race/ethnicity $(0=$ White; $1=$ non-White $)$, gender $(0=$ female; $1=$ male $)$, age, parental status $(0=$ no children; $1=1+$ children $)$, marital status ( $0=$ not married; $1=$ married $)$, employment status $(0=$ not employed; $1=$ employed full or part time $)$, years of education, birth order $(0=$ older than sibling; $1=$ younger than sibling), dyadic gender composition $(0=$ same gender; $1=$ mixed gender), sibling age difference, sibling parental status $(0=$ no children; $1=1+$ children $)$, sibling marital status $(0=$ not married; $1=$ married), sibling years of education, sibling relatedness $(0=$ not full biological siblings; 1 = full biological siblings), sibling residence distance, sibling conflict, sibling closeness, recalled maternal PDT, and recalled paternal PDT. All continuous variables were grand mean centered except for recalled differential treatment variables, which were centered at equal treatment. In the second step, we entered three dummy codes for the class membership groups (medium contact was the reference group). In the third step, we entered interactions between the dummy codes and the continuous variable of recalled maternal PDT. In the fourth step, recalled maternal PDT was omitted, and interactions with recalled paternal PDT (continuous variable) were included. Because the individual coefficients and corresponding $t$ tests for dummy codes in an OLS regression reflect differences between particular groups and the reference group, adding blocks of the interactions of recalled maternal PDT separately from recalled paternal PDT allowed for examination of the overall interaction of class membership with recalled PDT from each parent separately. We also assessed the normality of life satisfaction, self-rated health, recalled maternal PDT and recalled paternal PDT (Supplementary Table 3).

Results for life satisfaction (Table 2; Supplementary Table 4 contains the full information) revealed several main effects in the first step. In particular, those who were not White $(b=0.66, S E=.28, p<.05, \beta=.10)$, were older $(b=0.06, S E=.02, p<.001, \beta=.19)$, had children $(b=0.69$, $S E=.23, p<.01, \beta=.13)$, were married $(b=0.92, S E=.19$, $p<.001, \beta=.21)$, were employed $(b=0.41, S E=.20, p$ $<.05, \beta=.10)$, and felt closer to their sibling $(b=0.23$, $S E=.09, p<.05, \beta=.11)$, reported higher life satisfaction. In Step 2, the change in $R^{2}$ suggested that the addition of class membership accounted for a significant amount of the variation. Specifically, those in the high communication class reported significantly higher life satisfaction than the other classes. There were no other differences among the 
Table 2. Summary of Ordinary Least Squares Regression Testing Variables Associated with Life Satisfaction $(N=491)$

\begin{tabular}{|c|c|c|c|c|c|c|}
\hline \multirow[b]{2}{*}{ Variables } & \multicolumn{3}{|c|}{ Step 1} & \multicolumn{3}{|c|}{ Step 2} \\
\hline & $b$ & $S E$ & $\beta$ & $b$ & $S E$ & $\beta$ \\
\hline Intercept & $5.01 * * *$ & .76 & & $4.72 * * *$ & .78 & \\
\hline Recalled Maternal PDT & 0.16 & .12 & .06 & 0.16 & .12 & .06 \\
\hline Recalled Paternal PDT & -0.02 & .12 & -.01 & 0.00 & .12 & .00 \\
\hline Low Class Membership & & & & 0.16 & .26 & .04 \\
\hline Traditional Class Membership & & & & 0.01 & .29 & .00 \\
\hline High Class Membership & & & & $0.95 * *$ & .29 & .16 \\
\hline Model $R^{2}$ & & .15 & & & .17 & \\
\hline$F$ for change in $R^{2}$ & & $3.83 * * *$ & & & $3.96 * *$ & \\
\hline
\end{tabular}

Note: Omitted controls are: if mother is living, if father is living, if parents ever divorced, race/ethnicity, gender, age, parental status, marital status, employment status, education level, birth order, dyadic gender composition, sibling age difference, sibling parental status, sibling marital status, sibling education level, sibling relatedness, sibling residence distance, sibling conflict, and sibling closeness. PDT = Parental differential treatment. Additionally, interactions between recalled maternal PDT and each communication group as well as paternal PDT and each communication group were not significant, and were omitted from the table. Medium contact was the reference for the communication groups. A complete version of this table can be found in online supplementary materials (Supplementary Table 7 ). $* p<.01, * * p<.001$.

other classes. Additionally, the change in $R^{2}$ for both Step 3 and Step 4 suggested that class membership did not interact with recalled maternal PDT nor recalled paternal PDT.

Analysis for self-rated health (Table 3; Supplementary Table 5 contains the full information) revealed several main effects. Those whose mother was living $(b=0.24, S E=.11$, $p<.05, \beta=.11)$, were employed $(b=0.31, S E=.10, p<.01$, $\beta=.14)$, or were farther in age from their sibling $(b=-0.02$, $S E=.01, p<.05, \beta=-.11)$, reported better health. The change in $R^{2}$ in Step 2 revealed that the addition of class membership did not account for a significant amount of variation. The change in $R^{2}$ for Step 3, however, suggested a significant interaction between class membership and recalled maternal PDT. Post hoc testing of the significant interaction revealed several important findings (Figure 2). For those in the low, medium, and traditional classes, there was no association between recalled maternal PDT and self-rated health. For those in the high communication class, there was a positive association $(b=0.76, S E=.21, p<.001, \beta=.57)$, such that those who recalled receiving favored treatment reported better health. We also used post hoc testing to examine mean differences among the classes at recalled less-favored status one standard deviation below equal treatment, recalled equal treatment, and recalled favored status one standard deviation above equal treatment. At recalled less-favored status and at recalled equal treatment, there were no differences in self-rated health among the classes. At recalled favored status, there were no differences among those in the low, medium, or traditional classes. Those in the high communication class, however, reported significantly better health than those in the low $(t(1)=3.06, p<.01)$, medium $(t(1)=2.90$, $p<.01)$, and traditional $(t(1)=3.68, p<.001)$ classes. The addition of the interaction of recalled paternal PDT and class membership in Step 4 was not significant.

We conducted further post hoc analyses for both life satisfaction and self-rated health because of the ways our sample differed from the general population. Specifically, we tested whether significant effects (class membership linked with life satisfaction; class membership $\times$ recalled maternal PDT for self-rated health) varied by the ways our sample differed from the general population (age, years of education, parental status, employment status, if the mother was living, and if the father was living). None of the findings were qualified by these variables.

\section{Discussion}

Baby Boomers are rapidly reaching the later years of life, and by 2030 nearly $20 \%$ of Americans will be over the age of 65 (Cohn \& Taylor, 2010). Many older adults experience a shrinking of social networks (Pinquart \& Sorensen, 2000; Wrzus, Hanel, Wagner, \& Neyer, 2013), which may potentially make siblings more prominent in their lives than the preceding decades (Bedford \& Avioli, 2012; Liao \& Carstensen, 2018). The current study examined, first, the different patterns of contact among siblings in later life, and second, if those patterns were linked to two aspects of well-being, life satisfaction, and self-rated health. Based on Life Course Theory (Elder et al., 2003), we anticipated that links between patterns of contact and well-being would vary based on recollections of childhood experiences. In particular, we hypothesized that patterns of high contact would be linked to greater well-being for those who recalled being favored by their mother or father, and linked to lower well-being for those who recalled receiving lessfavored treatment.

\section{Patterns of Contact}

Overall, our findings suggest that different patterns of contact exist among siblings in the later years. For the majority of our sample, contact levels among different mediums were fairly consistent. Those who had low contact in one 
Table 3. Summary of Ordinary Least Squares Regression Testing Variables Associated with Self-rated Health $(N=491)$

\begin{tabular}{|c|c|c|c|c|c|c|c|c|c|}
\hline \multirow[b]{2}{*}{ Variables } & \multicolumn{3}{|c|}{ Step 1} & \multicolumn{3}{|c|}{ Step 2} & \multicolumn{3}{|c|}{ Step 3} \\
\hline & $b$ & $S E$ & $\beta$ & $b$ & $S E$ & $\beta$ & $b$ & $S E$ & $\beta$ \\
\hline Intercept & $1.85 * * *$ & .39 & & $1.77 * * *$ & .41 & & $1.70 * * *$ & .40 & \\
\hline Mom Living & $0.24 *$ & .11 & .11 & $0.25 *$ & .11 & .12 & $0.23 *$ & .11 & .11 \\
\hline Recalled Maternal PDT (MPDT) & 0.10 & .06 & .08 & 0.10 & .06 & .08 & 0.19 & .11 & .14 \\
\hline Recalled Paternal Favoritism & -0.09 & .06 & -.06 & -0.08 & .06 & -.06 & -0.05 & .06 & -.04 \\
\hline Low Class Membership (Low) & & & & 0.10 & .14 & .04 & 0.12 & .13 & .05 \\
\hline Traditional Class Membership (Trad) & & & & -0.07 & .15 & -.02 & -0.04 & .15 & -.02 \\
\hline High Class Membership (High) & & & & 0.26 & .15 & .09 & 0.18 & .15 & .06 \\
\hline MPDT $\times$ Low & & & & & & & -0.20 & .13 & -.11 \\
\hline MPDT $\times$ Trad & & & & & & & -0.34 & .22 & -.08 \\
\hline MPDT $\times$ High & & & & & & & $0.57 *$ & .24 & .12 \\
\hline Model $R^{2}$ & & .08 & & & .09 & & & .12 & \\
\hline$F$ for change in $R^{2}$ & & $1.95 * *$ & & & 1.58 & & & $4.64 * *$ & \\
\hline
\end{tabular}

Note: Omitted controls are: if mother is living, if father is living, if parents ever divorced, race/ethnicity, gender, age, parental status, marital status, employment status, education level, birth order, dyadic gender composition, sibling age difference, sibling parental status, sibling marital status, sibling education level, sibling relatedness, sibling residence distance, sibling conflict, and sibling closeness. PDT = Parental differential treatment. Additionally, interactions between recalled paternal PDT and each communication group were not significant, and were omitted from the table. Medium contact was the reference for the communication groups. A complete version of this table can be found in online supplementary materials (Supplementary Table 8). $* p<.05, * p<.01, * * p<.001$.

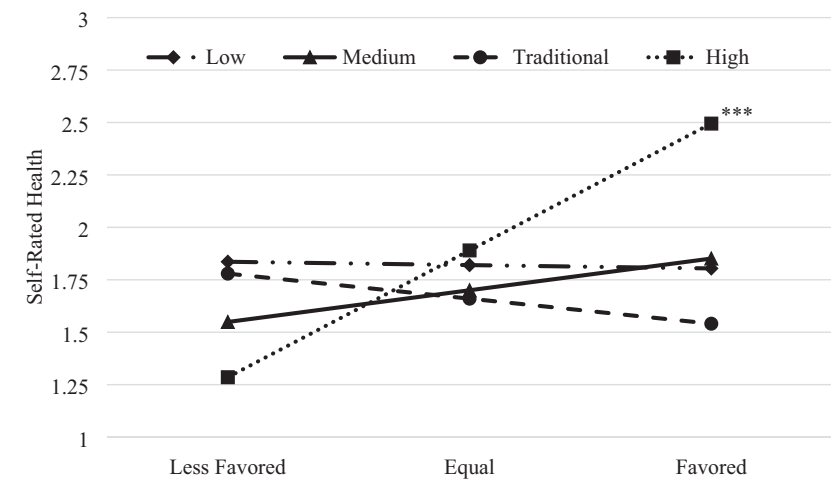

Figure 2. The association between recalled maternal parental differential treatment and self-rated health as moderated by pattern of sibling communication. ${ }^{* *} p<.001$.

domain also had low contact in the other domains. Similar patterns were observed for both medium and high levels of contact. Our finding of a class who communicated frequently through all measured mediums corroborates recent findings that many older adults are frequently using the internet and other technologies (Pew Research Center, 2017). Adults in the high communication class may be more comfortable with technology and find it helpful to communicate with a sibling. For example, those in the high class texted with their sibling on average over once a week, which was a little higher than any other means of communication. Those in the medium group also texted at similar or higher levels than other forms of contact. With the prevalence of smart phones among older adults (Pew Research Center, 2017) and the ease of texting, it is possible that for some older adults, texting will become an important way to connect with a sibling.
Beyond patterns marked by low, medium, and high contact, our analysis also revealed another group. These adults had medium to high contact with their sibling through more traditional means (in person and over the phone) but low contact through more modern means (email, texting, and social media). Given that our sample was collected online and our participants were significantly more educated than the general population, it is possible that our participants were comfortable with communicating via technological means, but their sibling is not. Future work will need to examine older adults' comfort levels with technology and how that contributes to their communication patterns with siblings. Perhaps helping older adults become comfortable with text messaging and social media may give them the ability to communicate more with siblings. Additionally, our sample was significantly younger than the national population of Baby Boomers/older adults, and analysis suggested that older participants were more likely to be in the traditional communication class. Thus, it is possible that among all older adults, a higher percentage fall into a traditional pattern of communication than was found in our data.

Beyond age, differences in employment, education, and fathers' living status may also play a role in our findings. Specifically, our sample was more educated, more likely to be working, and less likely to have a living father than the general population. Two of these factors would suggest that our sample has overall less contact than what may occur in the general population; thus, our sample may have net lower contact than the general population. Future studies will need nationally representative data because additional patterns of contact may exist if the overall levels of contact are higher. 


\section{Links with Well-Being}

Our hypothesis that patterns marked by higher contact would be linked to greater well-being was supported in regards to life satisfaction. Those in the high contact group reported significantly higher life satisfaction than those in the other groups, which is line with past studies that suggest social contact may promote the well-being of older adults (Gray, 2009; Pinquart \& Sorensen, 2000). Those in the traditional group had similar levels of in person and telephone contact compared to the high group, but still showed lower levels of life satisfaction. Although some recent work suggests that face to face communication is particularly important for adults (Teo et al., 2015) our work is more in line with the findings of Ferreira and colleagues (2015) which suggests that communication over texting or the internet has positive effects for older adults. Because our sample consists of active internet users who are more educated than the general population, they may see more salience in technological communication. Other work will need to replicate these findings including among less internet savvy older adults in representative data sets. Additionally, our data were cross-sectional, but future studies will need longitudinal data to examine the direction of effects; it is possible that those with high life satisfaction are more willing to communicate with siblings rather than the other way around.

There was also some support to the hypothesis that patterns of greater contact would be linked to self-rated health. Consistent with Life Course Theory (Bengston et al., 2005; Elder et al., 2003), this link was qualified by early life experience, or the recollection of PDT. For those in the high contact group, recalled maternal PDT was linked to selfrated health. Specifically, those who recalled favored status reported better health than those who recalled less-favored status. There was no association for those in the medium, traditional, or low contact groups. Frequent contact between siblings through a variety of mediums may provide more opportunities for the continuation of family patterns established at younger ages or the remuneration of shared memories that may serve as a reminder of differences in treatment.

There are, however, more nuances to the significant association between recalled PDT and self-rated health among those in the high communication class. Work from Suitor and colleagues (2017) on PDT in mid to later life and adults' well-being suggests that less-favored status is more likely detrimental than favored status is beneficial. In our post hoc analysis, those in the high contact class who recalled less-favored status did not differ in their health from others who recalled less-favored status but had less frequent contact with their sibling. Perhaps with concurrent differences in treatment, as examined by Suitor et al. (2017), being treated less favorably is damaging to well-being. With recollections of PDT from childhood, perhaps many adults have had time to work through and process negative feelings associated with less-favored treatment. In contrast, our findings suggest that those with recalled favored status in the high contact class reported better health than those in the other classes. Perhaps, for those who recall being favored, frequent contact reminds them of that preferential treatment, and even if it happens subconsciously, that reminder may promote positive selfconceptions that may translate to better health. As with life satisfaction, however, future studies will need to examine these processes longitudinally to establish the direction of effects.

Although we offered no hypotheses regarding differences in the roles of recalled maternal versus paternal PDT, our findings add to the literature of studies that have included these differences (Gilligan et al., 2013; Jensen et al., 2013). Overall, our findings suggest that recollections of maternal PDT are more salient than those of fathers. This is contrast to the findings of Gilligan et al. (2013), which suggest that paternal PDT is more salient. It is possible that concurrent PDT from fathers in mid to later life is more salient than that of mothers, but that recalled differences in treatment from mothers are more salient than recollections of paternal treatment. Future studies need to do more to delineate possible differences in recalled and concurrent PDT as each may have differing roles in adults' well-being.

\section{Conclusion}

Despite the strengths of the study, it was not without limitations. First, data were collected entirely online. As noted in the participants section, our sample differed from nationally representative data in several ways, including that they were younger, more educated, less likely to have children, more likely to be working, and more likely to have had a parent pass away. All of these factors likely change the likelihood of siblings maintaining contact and being an active part of one another's social networks. Additionally, our participants were experienced enough internet users to use MTurk means that they may be more technologically savvy than many older adults. Future work will need to replicate these findings in other samples not recruited through the internet. Additionally, we used single reporters. Sibling dynamics are inherently dyadic and the perceptions of one sibling may not match the other. Along those lines, we also only focused on participants' relationship with one sibling. Our participants, on average, had over two living siblings. Differences in how older adults communicate with different siblings are also important in understanding all the patterns of contact. Thus, future work will need to collect data from all the living siblings in the family. Lastly, the data were cross-sectional; so, we were unable to determine the direction of effects. Although we have argued that contact may promote or hinder well-being, it is possible that well-being promotes or hinders contact. Future work will need to collect longitudinal data and examine the direction 
of effects. Ultimately, longitudinal data will be necessary to determine the direction of effects.

Although this study was cross-sectional and could not assess causality, the findings make an important contribution in understanding sibling contact in later life. Siblings may serve as important members of social networks in mid to later life and promoting communication may help with life satisfaction and self-rated health for those who were favored as a child.

\section{Supplementary Material}

Supplementary data is available at The Journals of Gerontology, Series B: Psychological Sciences and Social Sciences online.

\section{Funding}

This work was supported by internal funding.

\section{Conflict of Interest}

None reported.

\section{Author Contributions}

A. C. Jensen contributed by aiding in data collection, analysis, and manuscript preparation. M. K. Nielson contributed by aiding in data collection and manuscript preparation. J. B. Yorgason contributed by helping with analysis and manuscript preparation.

\section{References}

Bedford, V. H. (1989). Understanding the value of siblings in old age. American Behavioral Scientist 33, 33-44. doi:10.1177/0002764289033001004

Bedford, V. H., \& Avioli, P. S. (2012). Sibling relationships from midlife to old age. In R. Blieszner \& V. H. Bedford (Eds.), Handbook of families and aging, 2nd ed. (pp. 125-151). Santa Barbara, CA.

Bengston, V. L., Elder, G. H. J., \& Putney, N. M. (2005). The life course perspective on ageing linked lives, timing, and history. In M. L. Johnson (Ed.), The Cambridge handbook of age and ageing (pp. 9-17). Cambridge: Cambridge University Press.

Blyth, D. A., \& Foster-Clark, F. S. (1987). Gender differences in perceived intimacy with different members of adolescents' social networks. Sex Roles, 17, 689-718. doi:10.1007/BF00287683

Bohannon, J. (2011). Human subject research. Social science for pennies. Science (New York, N.Y.), 334, 307. doi:10.1126/ science.334.6054.307

Boll, T., Ferring, D., \& Filipp, S. H. (2003). Perceived parental differential treatment in middle adulthood: Curvilinear relations with individuals' experienced relationship quality to sibling and parents. Journal of Family Psychology, 17, 472-487. doi:10.1037/0893-3200.17.4.472
Buhrmester, M., Kwang, T., \& Gosling, S. D. (2011). Amazon's mechanical Turk: A new source of inexpensive, yet highquality, data? Perspectives on Psychological Science, 6, 3-5. doi:10.1177/1745691610393980

Casler, K., Bickel, L., \& Hackett, E. (2013). Separate but equal? A comparison of participants and data gathered via Amazon's MTurk, social media, and face-to-face behavioral testing. Computers in Human Behavior, 29, 2156-2160. doi:10.1016/j. chb.2013.05.009

Caspi, A., Elder, G. H., \& Bem, D. J. (1988). Moving away from the world: Life-course patterns of shy children. Developmental Psychology, 24, 824-831. doi:10.1037/0012-1649.24.6.824

Cohn, D. \& Taylor, P. (2010). Baby Boomers approach 65 - glumly. Retrieved from http://www.pewsocialtrends.org/2010/12/20/ baby-boomers-approach-65-glumly/.

Daniels, D., \& Plomin, R. (1985). Differential experience of siblings in the same family. Developmental Psychology, 21, 747-760. doi:10.1037/0012-1649.21.5.747

Davey, A., Tucker, C. J., Fingerman, K., \& Savla, J. (2009). Within-family variability in representations of past relationships with parents. The Journals of Gerontology, Series B: Psychological Sciences and Social Sciences, 64, 125-136. doi:10.1093/geronb/gbn001

Diener, E., Gohm, C. L., Suh, E., \& Oishi, S. (2000). Similarity of the relations between marital status and subjective well-being across cultures. Journal of Cross-Cultural Psychology, 31, 419-436. doi:10.1177/0022022100031004001

Elder, G. H., Johnson, M. K., \& Crosnoe, R. (2003) The emergence and development of life course theory. In J. T. Mortimer, \& M. J. Shanahan (Eds.), Handbook of the Life Course. Handbooks of Sociology and Social Research. Boston, MA: Springer.

Feinberg, M. E., Solmeyer, A. R., \& McHale, S. M. (2012). The third rail of family systems: Sibling relationships, mental and behavioral health, and preventive intervention in childhood and adolescence. Clinical Child and Family Psychology Review, 15, 43-57. doi:10.1007/s10567-011-0104-5

Ferreira, S., Torres, A., Mealha, O., \& Velso, A. (2015). Training effects on older adults in information and communication technologies considering psychosocial variables. Educational Gerontology, 41, 482-493. doi:10.1080/03601277.2014.994351

Gilligan, M., Suitor, J. J., Kim, S., \& Pillemer, K. (2013). Differential effects of perceptions of mothers' and fathers' favoritism on sibling tension in adulthood. The Journals of Gerontology, Series B: Psychological Sciences and Social Sciences, 68, 593-598. doi:10.1093/geronb/gbt039

Gray, A. (2009). The social capital of older people. Ageing \& Society, 29, 5-31. doi:10.1017/S0144686X08007617

Halliwell, D., Wenzel Egan, K. A., \& Howard, E. L. (2017). Flying in a $\mathrm{V}$ formation: Themes of (in)equity, reality, and togetherness in adult siblings' narrative explanations of shared parental caregiving. Journal of Applied Communication Research, 45, 256273. doi:10.1080/00909882.2017.1320574

Ipeirotis, P. G. (2010). Demographics of Mechanical Turk. Center for Digital Economy Research Working Papers, 10. Retrieved from http://crowdsourcing-class.org/readings/downloads/platform/demographics-of-mturk.pdf.

Jensen, A. C., Whiteman, S. D., \& Fingerman, K. L. (2018). “Can't live with them, can't live without them:" Life transitions and 
sibling relationships in young adulthood. Journal of Family Psychology, 32, 385-395. doi:10.1037/fam0000361

Jensen, A. C., Whiteman, S. D., Fingerman, K. L., \& Birditt, K. S. (2013). "Life Still Isn't Fair": Parental differential treatment of young adult siblings. Journal of Marriage and the Family, 75, 438-452. doi:10.1111/jomf.12002

Liao, H. W., \& Carstensen, L. L. (2018). Future time perspective: Time horizons and beyond. Geropsych, 31, 163-167. doi:10.1024/1662-9647/a000194

Litwin, H., \& Stoeckel, K. J. (2013). Social networks and subjective wellbeing among older Europeans: Does age make a difference? Ageing of Society, 33, 1263-1281. doi:10.1017/ S0144686X12000645

Milevsky, A. (2005). Compensatory patterns of sibling support in emerging adulthood: Variations in loneliness, self-esteem, depression, and life satisfaction. Journal of Social and Personal Relationships, 22, 743-755. doi:10.1177/0265407505056447

Mossey, J. M., \& Shapiro, E. (1982). Self-rated health: A predictor of mortality among the elderly. American Journal of Public Health, 72, 800-808. doi:10.2105/ajph.72.8.800

Munnell, A. H. (2015). The average retirement age - an update. The Center for Retirement Research at Boston College, Briefs. Retrieved from http://crr.bc.edu/wp-content/uploads/2015/03/IB_15-4.pdf.

Muthén, L. K., \& Muthén, B. O. (2017). Mplus Version 8 User's Guide. Los Angeles, CA: Author.

Peng, S., Suitor, J. J., \& Gilligan, M. (2018). The long arm of maternal differential treatment: Effects of recalled and current favoritism on adult children's psychological well-being. The Journals of Gerontology, Series B: Psychological Sciences and Social Sciences, 73, 1123-1132. doi:10.1093/geronb/gbw105

Pew Research Center. (2017). Internet/Broadband fact sheet. Retrieved from http://www.pewinternet.org/fact-sheet/internet-broadband/

Pinquart, M., \& Sörensen, S. (2000). Influences of socioeconomic status, social network, and competence on subjective well-being in later life: A meta-analysis. Psychology and Aging, 15, 187224. doi:10.1037/0882-7974.15.2.187

Rouse, S. V. (2015). A reliability analysis of mechanical Turk data. Computers in Human Behavior, 43, 304-307. doi:10.1016/j. chb.2014.11.004
Scholte, R. H., Engels, R. C. M. E., de Kemp, R. A. T., Harakeh, Z., \& Overbeek, G. (2007). Differential parental treatment, sibling relationships and delinquency in adolescence. Journal of Youth and Adolescence, 36, 661-671. doi:10.1007/ s10964-006-9155-1

Siordia, C., \& Leyser-Whalen, O. (2017). Average age at last live birth in 'lucky few' and 'late baby boom' birth cohorts in the United States. Journal of Family Studies, 23, 296-308. doi:10.10 80/13229400.2015.1106338

Silverstein, M., Gans, D., \& Yang, F. M. (2006). Intergenerational support to aging parents: The role of norms and needs. Journal of Family Issues, 27, 1068-1084. doi:10.1177/ 0192513X06288120

Stocker, C. M., \& McHale, S. M. (1992). The nature and family correlates of preadolescents' perceptions of their sibling relationships. Journal of Personal and Social Relationships, 9, 179-195. doi:10.1177/0265407592092002

Suitor, J. J., Gilligan, M., Peng, S., Jung, J. H., \& Pillemer, K. (2017). Role of perceived maternal favoritism and disfavoritism in adult children's psychological well-being. The Journals of Gerontology, Series B: Psychological Sciences and Social Sciences, 72, 10541066. doi:10.1093/geronb/gbv089

Suitor, J. J., Sechrist, J., Plikuhn, M., Pardo, S. T., Gilligan, M., \& Pillemer, K. (2009). The role of perceived maternal favoritism in sibling relations in midlife. Journal of Marriage and the Family, 71, 1026-1038. doi:10.1111/j.1741-3737.2009.00650.x

Teo, A. R., Choi, H., Andrea, S. B., Valenstein, M., Newsom, J. T., Dobscha, S. K., \& Zivin, K. (2015). Does mode of contact with different types of social relationships predict depression in older adults? Evidence from a nationally representative survey. Journal of the American Geriatrics Society, 63, 2014-2022. doi:10.1111/ jgs. 13667

White, L. (2001). Sibling relationships over the life course: A panel analysis. Journal of Marriage and Family, 63, 555-568. doi:10.1111/j.1741-3737.2001.00555.x

Wrzus, C., Hänel, M., Wagner, J., \& Neyer, F. J. (2013). Social network changes and life events across the life span: A metaanalysis. Psychological Bulletin, 139, 53-80. doi:10.1037/ a0028601 